P. W. Sy and T. Sunada

Nagoya Math. J.

Vol. 125 (1992), 141-150

\title{
DISCRETE SCHRÖDINGER OPERATORS ON A GRAPH
}

\author{
POLLY WEE SY* AND TOSHIKAZU SUNADA
}

In this paper, we study some spectral properties of the discrete Schrödinger operator $-\Delta+q$ defined on a locally finite connected graph with an automorphism group whose orbit space is a finite graph.

The discrete Laplacian and its generalization have been explored from many different viewpoints (for instance, see [2] [4]). Our paper discusses the discrete analogue of the results on the bottom of the spectrum established by T. Kobayashi, K. Ono and T. Sunada [3] in the Riemannianmanifold-setting.

\section{§1. Discrete Laplacians}

Let $X=(V, E)$ be a locally finite connected graph without loops and multiple edges. Here $V$ and $E$ are, respectively, the set of vertices and the set of unoriented edges of $X$. In a natural manner, $X$ is regarded as a one-dimensional $\mathrm{CW}$ complex. We assign a positive weight to each vertex and also to each edge by giving mappings $m: V \rightarrow \mathbb{R}_{+}$and $w: E$ $\rightarrow \mathbb{R}_{+}$. Let $C_{0}(V)$ and $C_{0}(E)$ be the space of all complex-valued functions on $V$ and $E$ with finite support, respectively. Define inner products on $C_{0}(V)$ and $C_{0}(E)$ by

$$
\begin{aligned}
& \langle f, g\rangle=\sum_{x \in V} f(x) \overline{g(x)} m(x) \\
& \langle\omega, \eta\rangle=\sum_{e \in E} \omega(e) \overline{\eta(e)} w(e) .
\end{aligned}
$$

The completions of $C_{0}(V)$ and $C_{0}(E)$ with respect to those inner products will be denoted by $L^{2}(V)$ and $L^{2}(E)$, respectively.

Each edge has two orientations. We use the symbol $E^{\text {or }}$ to represent the set of all oriented edges, so that forgetting orientation yields a twoto-one map $p: E^{\text {or }} \rightarrow E$. Reversing orientation gives rise to an involution on $E^{\text {or }}$, which we denote by $e \mapsto \bar{e}$. We shall use the same symbol $w$ for

Received December 13, 1989.

* Research was supported in part by Japan Society for the Promotion of Science. 
the composition $w \circ p$, which is a function on $E^{\text {or }}$. For an oriented edge $e, \mathfrak{o}(e)$ and $t(e)$ denote the origin and terminus point of $e$, respectively. Let $\mathcal{O}_{x}=\left\{e \in E^{\text {or }} ; \mathfrak{o}(e)=x\right\}$.

We fix an orientation on each edge by giving a subset $E_{0}$ of $E^{\text {or }}$ such that $E^{\text {or }}=E_{0} \cup \bar{E}_{0}$ (disjoint) and we identify $E_{0}$ with $E$ by the map $p$. Define the operator $d: C_{0}(V) \rightarrow C_{0}(E)$ by

$$
d f(e)=f(\mathrm{t}(e))-f(\mathfrak{o}(e)),
$$

which is a natural analogue of the exterior derivation on a manifold.

A simple calculation gives the following formula for the formal adjoint $d^{*}$ of $d$ :

$$
d^{*} \omega(x)=m(x)^{-1}\left\{\sum_{\substack{e \in E_{0} \\ \mathrm{t}(e)=x}} \omega(e) w(e)-\sum_{\substack{e \in E_{0} \\ 0(e)=x}} \omega(e) w(e)\right\} .
$$

The discrete Laplacian $\Delta=\Delta_{X}$ is now defined by

$$
\Delta f(x)=-d^{*} d f(x)=m(x)^{-1}\left\{\sum_{e \in \mathscr{o}_{x}} f(\mathrm{t}(e)) w(e)-\left(\sum_{e \in \mathcal{O}_{x}} w(e)\right) f(x)\right\} .
$$

Note that $\Delta$ is independent of the choice of orientation on edges.

Remark 1. Let $h: V \rightarrow \mathbb{R}$ be a function defined by

$$
h(x)=(1 / m(x)) \sum_{e \in \mathscr{o}_{x}} w(e) .
$$

Then the operator $\Delta$ is bounded as an operator acting in $L^{2}(V)$ if and only if $h$ is bounded. For the sake of completeness, we shall give a proof. Suppose that $h$ is bounded. Then for any $f \in C_{0}(V)$,

$$
\begin{aligned}
\|d f\|^{2} & \leq 2 \sum_{e \in E_{0}}\left(|f(\mathrm{t}(e))|^{2}+|f(\mathfrak{o}(e))|^{2}\right) w(e) \\
& =2\left\{\sum_{x \in V} \sum_{\substack{e \in E_{0} \\
t(e)=x}}|f(\mathrm{t}(e))|^{2} w(e)+\sum_{\substack { x \in V \\
\begin{subarray}{c}{e \in E_{0}^{\prime} \\
0(e)=x{ x \in V \\
\begin{subarray} { c } { e \in E _ { 0 } ^ { \prime } \\
0 ( e ) = x } }\end{subarray}}|f(\mathfrak{o}(e))|^{2} w(e)\right\} \\
& =2\left\{\sum_{x \in V}|f(x)|^{2}\left(\sum_{c \in \mathcal{O}_{x}} w(e)\right)\right\} \\
& \leq c\|f\|^{2}
\end{aligned}
$$

where $c=2 \sup _{x \in V}\left\{(1 / m(x)) \sum_{e \in o_{x}} w(e)\right\}$. Thus $\Delta$ is bounded. Conversely, assume that $\Delta$ is bounded. If $h$ is unbounded, then for every positive real number $K$, there is an $x \in V$ such that $(1 / m(x)) \sum_{e \in \theta_{x}} w(e) \geq K$. We see that $\left\|d \delta_{x}\right\|^{2}=\sum_{e \in 0_{x}} w(e) \geq K m(x)=K\left\|\delta_{x}\right\|^{2}$, where $\delta_{x}(y)$ equals 1 when $y=x$ and zero elsewhere. It follows that $\left\|\Delta \delta_{x}\right\|\left\|\delta_{x}\right\| \geq\left|\left(d^{*} d \delta_{x}, \delta_{x}\right)\right|=\left\|d \delta_{x}\right\|^{2}$ $\geq K\left\|\delta_{x}\right\|^{2}$. Thus $\Delta$ is unbounded. This contradicts our hypothesis that $\Delta$ is bounded.

Remark 2. The discrete Laplacian defined above is a bit generalized 
one of [2].

\section{§2. Bottom of the spectrum}

Let $M=(V, E)$ be a finite connected graph, and let $\pi: X \rightarrow M$ be a normal covering map as $\mathrm{CW}$ complexes with the covering transformation group $\Gamma$. The covering space $X$ has a graph structure $(\tilde{V}, \tilde{E})$ such that $\pi$ is a morphism of graphs. Then $\Gamma$ acts freely on $\tilde{E}$ and $\tilde{V}$ and $\Gamma \backslash \tilde{E} \simeq E$, $\Gamma \backslash \tilde{V} \simeq V$. We assume that $M$ has weights on vertices and edges. The weights on vertices and edges of $X$ are naturally assigned by using the map $\pi$ so that they are left invariant under the $\Gamma$-action. If we fix orientation on edges of $M$, then the induced one on $\tilde{E}$ is preserved by the $\Gamma$-action. Take any real-valued function $q_{M} \in C(V)$. We see that $q=q_{M} \circ \pi$ is invariant under the $\Gamma$-action. Since $M$ is finite, $H_{M}=-\Delta_{M}+q_{M}$ is identified with a hermitian matrix of finite size and its spectrum consists of real eigenvalues.

The operator $H_{X}=-\Delta_{X}+q$ is just the lift of the operator $H_{M}$ on $M$ by the map $\pi$ and is therefore bounded (see Remark 1) and self-adjoint. We denote by $\lambda_{0}(H)$ the greatest lower bound of the spectrum of a selfadjoint operator $H$. Note that $\lambda_{0}\left(H_{M}\right)$ is just the minimal eigenvalue of $H_{M}$.

Lemma 1. $\lambda_{0}\left(H_{M}\right)$ is simple and has a positive eigenfunction.

Proof. Let $V=\{1, \cdots, n\}$. For $1 \leq i \leq n$, set

$$
\begin{aligned}
\varphi_{i}(x) & =\frac{1}{\sqrt{m(i)}} & & \text { if } x=i \\
& =0 & & \text { otherwise. }
\end{aligned}
$$

Then $\left\{\varphi_{i}\right\}$ is an orthonormal basis of $L^{2}(V)$. Let $A=\left(a_{i j}\right)$ be the matrix of $\Delta_{M}$ with respect to this basis. If $(i, j)$ is an edge of $M$ with $i \neq j$, then $a_{i j}=\left(\Delta_{M} \varphi_{j}, \varphi_{i}\right)=(1 / \sqrt{m(i) m(j)}) w(i, j)$. Hence the off-diagonal entries of the matrix $A$ are nonnegative real numbers. Let $A^{\prime}=\left(a_{i j}^{\prime}\right)$ be the matrix with $a_{i j}^{\prime}=a_{i j}$ for $i \neq j$ and $a_{i i}^{\prime}=0$. Since $M$ is connected, the matrix $A^{\prime}$ is irreducible. Thus the operator $\Delta_{M}-q_{M}$ has the form $A^{\prime}+D$, where $D$ is a diagonal matrix with entries $d_{i i} \in \mathbb{R}$. The facts that the maximal eigenvalue $-\lambda_{0}\left(A^{\prime}+D\right)\left(=-\lambda_{0}\left(H_{M}\right)\right)$ is simple and there exists a positive eigenfunction associated with it, follow readily by applying the PerronFrobenius Theorem [5] to the matrix $A^{\prime}+D+x I$ for large enough $x \in \mathbb{R}$.

Theorem 1. $\lambda_{0}\left(H_{M}\right) \leq \lambda_{0}\left(H_{X}\right)$. The equality holds if and only if the covering transformation group $\Gamma$ is amenable. 
To prove this, we will employ a representation-theoretic technique. We fix orientation $\tilde{E}_{0}$ on $\tilde{E}$ induced from an orientation of edges of $M$. We also identify $\tilde{E}_{0}$ with $\tilde{E}$.

Let $\rho$ be a unitary representation of $\Gamma$ on a Hilbert space $W$ and $L_{\rho}^{2}(V)=\{s: \tilde{V} \mapsto W ; s(\sigma x)=\rho(\sigma) s(x)$ for all $x \in \tilde{V}$ and $\sigma \in \Gamma\}$ with the natural inner product

$$
\left\langle s_{1}, s_{2}\right\rangle=\sum_{x \in \mathscr{Q}_{V}}\left\langle s_{1}(x), s_{2}(x)\right\rangle_{W} m(x),
$$

where $\mathscr{D}_{V}$ is a finite fundamental subset in $\tilde{V}$ for the $\Gamma$-action; i.e., $\mathscr{D}_{V}$ is a subset of $\tilde{V}$ such that for every $x \in \tilde{V}$, there exists a unique pair $\left(\sigma, x^{\prime}\right) \in \Gamma \times \mathscr{D}_{V}$ satisfying $\sigma x=x^{\prime}$. Note that $\tilde{V}=\bigcap_{\gamma \in \Gamma} \gamma \mathscr{D}_{V}$ and $\gamma \mathscr{D}_{V} \cap \mathscr{D}_{V}$ $=\phi$ for $\gamma \neq \mathrm{id}$. One can easily check that the inner product is independent of the choice of $\mathscr{D}_{V}$. Let $L_{\rho}^{2}(E)=\{\varphi: \tilde{E} \mapsto W ; \varphi(\sigma e)=\rho(\sigma) \varphi(e)$ for all $e \in \tilde{E}$ and $\sigma \in \Gamma\}$ with the following inner product

$$
\left\langle\varphi_{1}, \varphi_{2}\right\rangle=\sum_{e \in \mathscr{\Phi}_{E}}\left\langle\varphi_{1}(e), \varphi_{2}(e)\right\rangle_{W} w(e),
$$

where $\mathscr{D}_{E}$ is a finite fundamental subset in $\tilde{E}$ for the $\Gamma$-action. This definition also does not depend on the choice of $\mathscr{D}_{E}$.

The bounded operator $d_{\rho}: L_{\rho}^{2}(V) \rightarrow L_{\rho}^{2}(E)$ is defined by

$$
d_{\rho} s(e)=s(\mathrm{t}(e))-s(\mathfrak{o}(e)) .
$$

Lemma 2. The adjoint operator of $d_{o}$ is given by

$$
\left(d_{\rho}^{*} \varphi\right)(x)=m(x)^{-1}\left(\sum_{\substack{e \in E_{0} \\ t(e)=x}} \varphi(e) w(e)-\sum_{\substack{e \in \tilde{E}_{0} \\ 0(e)=0}} \varphi(e) w(e)\right) .
$$

Proof. First note that the correspondences

$$
\begin{array}{ll}
d_{1}: s \longmapsto \varphi_{1} & \varphi_{1}(e)=s(\mathfrak{t}(e)) \\
d_{2}: s \longmapsto \varphi_{2} & \varphi_{2}(e)=s(\mathfrak{v}(e))
\end{array}
$$

give rise to operators of $L_{\rho}^{2}(V)$ into $L_{\rho}^{2}(E)$, and $d_{\rho}=d_{1}-d_{2}$. Let $\mathscr{D}_{V}$ be a fundamental set in $\tilde{V}$, and put

$$
\mathscr{D}_{E}=\left\{e \in \tilde{E}_{0} ; t(e) \in \mathscr{D}_{V}\right\} \text {. }
$$

Then $\mathscr{D}_{E}$ is a fundamental set in $\tilde{E}=\tilde{E}_{0}$, and

$$
\begin{aligned}
\left\langle d_{1} s, \varphi\right\rangle & =\sum_{e \in \mathscr{\mathscr { S }}_{E}}\langle s(\mathrm{t}(e)), \varphi(e)\rangle_{W} w(e) \\
& =\sum_{x \in \mathscr{D}_{V}} \sum_{\substack{e \in \tilde{E}_{0} \\
t(e)=x}}\langle s(x), \varphi(e)\rangle_{W} w(e) .
\end{aligned}
$$


Thus we have

$$
d_{1}^{*} \varphi(x)=m(x)^{-1} \sum_{\substack{e \in E_{0} \\ \mathfrak{t}(e)=x}} \varphi(e) w(e)
$$

Similarly, we obtain

$$
d_{2}^{*} \varphi(x)=m(x)^{-1} \sum_{\substack{e \in \mathbb{E}_{0} \\ 0(e)=x}} \varphi(e) w(e)
$$

This completes the proof.

The Laplacian $\Delta_{\rho}$ acting on $L_{\rho}^{2}(V)$ is now defined by $-d_{\rho}^{*} d_{\rho}$ which is equal to

$$
\Delta_{p} s(x)=m(x)^{-1}\left\{\sum_{e \in \mathcal{O}_{x}} s(t(e)) w(e)-\left(\sum_{e \in \mathcal{O}_{x}} w(e)\right) s(x)\right\} .
$$

The twisted discrete Schrödinger operator is then defined as the selfadjoint operator $H_{\rho}=-\Delta_{\rho}+q$.

LEMMA 3. If $\rho$ is the right regular representation of $\Gamma$, then $\left(H_{\rho}, L_{\rho}^{2}(V)\right)$ is unitarily equivalent to $\left(H_{X}, L^{2}(\tilde{V})\right)$; and if $\rho$ is the trivial representation 1 , then $\left(H_{\rho}, L_{\rho}^{2}(V)\right)$ is unitarily equivalent to $\left(H_{M}, L^{2}(V)\right)$.

Proof. Let $W=L^{2}(\Gamma)=\left\{\varphi:\left.\Gamma \rightarrow \mathbb{C}\left|\sum_{\sigma \in \Gamma}\right| \varphi(\sigma)\right|^{2}<\infty\right\}$ and $\rho$ be the right regular representation $\rho_{r}$ of $\Gamma$ on $W$. From now on, we simply write $\rho$ for $\rho_{r}$. To prove that $H_{\rho}$ and $H_{X}$ are unitarily equivalent to each other, we have to show that there exists a unitary map $\Phi: L^{2}(\tilde{V}) \mapsto L_{\rho}^{2}(V)$ such that $H_{\rho} \circ \Phi=\Phi \circ H_{X}$.

Define the map $\Phi: C_{0}(\tilde{V}) \mapsto L_{\rho}^{2}(V)$ by

$$
\Phi(f)=s,
$$

where the function $s$ is defined to be $s(x)(\sigma)=f(\sigma x)$ for $x \in \tilde{V}, \sigma \in \Gamma$. One can check that $s(\mu x)=\rho(\mu) s(x)$ for any $\mu \in \Gamma, x \in \tilde{V}$. By the definition of fundamental set, we have

$$
\begin{aligned}
\|s\|^{2} & =\sum_{x \in \mathscr{Q}_{V}}\|s(x)\|_{W}^{2} m(x) \\
& =\sum_{x \in \mathscr{Q}_{V}} \sum_{\sigma \in \Gamma}|f(\sigma x)|^{2} m(x) \\
& =\|f\|^{2}
\end{aligned}
$$

for any $f \in C_{0}(\tilde{V})$. Thus $s \in L_{\rho}^{2}(V)$. Hence the map $\Phi$ is extended uniquely to an isometry of $L^{2}(\tilde{V})$ into $L_{\rho}^{2}(V)$.

Next, we claim that $\Phi$ is onto. Take any $s \in L_{\rho}^{2}(V)$, define $f: \tilde{V} \rightarrow \mathbb{C}$ 
by $f(x)=s(x) 1$, where 1 is the identity element of $\Gamma$. Since

$$
\begin{aligned}
\sum_{x \in \tilde{V}}|f(x)|^{2} m(x) & =\sum_{x \in \tilde{V}}|s(x) 1|^{2} m(x) \\
& =\sum_{x \in \mathscr{\Phi}_{V}} \sum_{\sigma \in \Gamma}|\rho(\sigma) s(x) 1|^{2} m(x) \\
& =\sum_{x \in \mathscr{\Phi}_{V}} \sum_{\sigma \in \Gamma}|s(x) \sigma|^{2} m(x) \\
& =\sum_{x \in \mathscr{Q}_{V}}\|s(x)\|^{2} m(x),
\end{aligned}
$$

therefore $f \in L^{2}(\tilde{V})$. Put $s^{\prime}=\Phi(f)$. Then $s^{\prime}(x)(\sigma)=f(\sigma x)=s(\sigma x) 1=[\rho(\sigma) s(x)] 1$ $=s(x)(\sigma)$ for every $x \in \tilde{V}$ and $\sigma \in \Gamma$. Hence $\Phi(f)=s^{\prime}=s$.

For any $f \in L^{2}(\tilde{V})$, we have

$$
\begin{aligned}
& \left(\left\{H_{\rho} \circ \Phi(f)\right\}(x)\right)(\sigma)=\left(\left\{H_{\rho} \circ s(x)\right\}(\sigma)\right) \\
& \quad=-\frac{1}{m(x)}\left\{\sum_{e \in o_{x}} s(\mathrm{t}(e)) \sigma w(e)-\left(\sum_{e \in \mathcal{O}_{x}} w(e)\right) s(x) \sigma\right\}+q(x) s(x) \sigma \\
& \quad=-\frac{1}{m(x)}\left\{\sum_{e \in o_{x}} f(\sigma \mathrm{t}(e)) w(e)-\left(\sum_{e \in o_{x}} w(e)\right) f(\sigma x)\right\}+q(x) f(\sigma x) \\
& \quad=-\frac{1}{m(x)}\left\{\sum_{e \in \sigma_{\sigma x}} f(\mathrm{t}(e)) w(e)-\left(\sum_{e \in \mathcal{O}_{x}} w(e)\right) f(\sigma x)\right\}+q(\sigma x) f(\sigma x) \\
& \quad=H_{X} f(\sigma x) \\
& \quad=\left(\left\{\Phi \circ H_{X}(f)\right\}(x)\right) \sigma .
\end{aligned}
$$

This proves the first part of the theorem.

The second part of the theorem is easy to prove.

The Kazhdan distance $\delta(\rho, \mathbf{1})$ (or $\left.\delta_{A}(\rho, \mathbf{1})\right)$ between $\rho$ and $\mathbf{1}$ is defined by

$$
\delta(\rho, \mathbf{1})=\inf _{\substack{v \in w \\\|r\|=1}} \sup _{\sigma \in A}\|\rho(\sigma) v-v\|,
$$

where $A$ is a fixed finite set of generators of $\Gamma$. The following lemma shows that the distance does not depend essentially on the choice of $A$.

Lemma 4. Suppose that $A$ and $B$ are any finite sets of generators of $\Gamma$. Then there exist positive constants $k_{1}$ and $k_{2}$ such that

$$
k_{1} \delta_{B}(\rho, \mathbf{1}) \leq \delta_{A}(\rho, \mathbf{1}) \leq k_{2} \delta_{B}(\rho, \mathbf{1}) .
$$

Proof. Let $C=A \cup B$. Choose an integer $N$ large enough such that every $\sigma \in C$ can be expressed as

$$
\sigma=\mu_{1} \mu_{2} \cdots \mu_{n},
$$

where $\mu_{i} \in A$ and $n \leq N$. Then 


$$
\begin{aligned}
\|\rho(\sigma) v-v\| \leq & \left\|\rho\left(\mu_{1}\right) \cdots \rho\left(\mu_{n}\right) v-\rho\left(\mu_{1}\right) \cdots \rho\left(\mu_{n-1}\right) v\right\| \\
& +\left\|\rho\left(\mu_{1}\right) \cdots \rho\left(\mu_{n-1}\right) v-v\right\| \\
\leq & \left\|\rho\left(\mu_{n}\right) v-v\right\|+\left\|\rho\left(\mu_{1}\right) \cdots \rho\left(\mu_{n-1}\right) v-v\right\| \\
\leq & \sum_{i=1}^{n}\left\|\rho\left(\mu_{i}\right) v-v\right\| \\
\leq & N \sup _{n \in A}\|\rho(\mu) v-v\| .
\end{aligned}
$$

It follows that $\delta_{A} \geq c_{1} \delta_{C}$ for some constant $c_{1}$. Similarly, one can also show that $\delta_{B} \geq c_{2} \delta_{C}$ for some constant $c_{2}$. On the other hand, since $A, B \subset C$, we have $\delta_{C} \geq \delta_{A}, \delta_{B}$. These inequalities together prove the result.

To prove Theorem 1, it suffices to establish the following Theorem (cf. [4] [7]). For, in the next theorem, when $\rho$ is the right regular representation $\rho_{r}$, Theorem 1 follows from the fact that $\delta\left(\rho_{r}, \mathbf{1}\right)=0$ if and only if $\Gamma$ is amenable.

THEOREM 2. There exist positive constants $c_{1}$ and $c_{2}$ such that

$$
c_{1} \delta(\rho, \mathbf{1})^{2} \leq \lambda_{0}\left(H_{\rho}\right)-\lambda_{0}\left(H_{1}\right) \leq c_{2} \delta(\rho, \mathbf{1})^{2}
$$

for all $\rho$. In particular, $\lambda_{0}\left(H_{\rho}\right)=\lambda_{0}\left(H_{1}\right)$ if and only if $\delta(\rho, \mathbf{1})=0$.

Proof. Note that

$$
\lambda_{0}\left(H_{\rho}\right)=\inf _{s \in L_{\rho}^{2}(V)} \frac{\left\langle H_{\rho} s, s\right\rangle}{\|s\|^{2}} .
$$

By Lemma 1, we may take a positive solution $f \in L^{2}(V)$ to the equation $H_{M} f=\lambda_{0}\left(H_{1}\right) f$. We have

$$
\begin{aligned}
\left\langle\Delta_{\rho}(f s), f s\right\rangle= & \sum_{x \in \mathscr{Q}_{V}}\left\langle\sum_{e \in \mathscr{o}_{x}} f(\mathrm{t}(e)) s(\mathrm{t}(e)) w(e)\right. \\
& \left.-\left(\sum_{e \in o_{x}} w(e)\right) f(x) s(x), f(x) s(x)\right\rangle .
\end{aligned}
$$

Substituting the following equality

$$
\sum_{e \in \mathcal{O}_{x}} w(e) f(x)=\lambda_{0}\left(H_{1}\right) f(x) m(x)-q(x) f(x) m(x)+\sum_{e \in \mathscr{o}_{x}} f(\mathrm{t}(e)) w(e)
$$

into (2.1), we obtain

$$
\begin{gathered}
\left\langle\Delta_{\rho}(f s), f s\right\rangle=\sum_{x \in \mathscr{Q}_{V}}\left\langle\sum_{e \in o_{x}} f(\mathfrak{t}(e))(s(\mathfrak{t}(e))-s(x)) w(e), f(x) s(x)\right\rangle \\
-\lambda_{0}\left(H_{1}\right)\langle f s, f s\rangle+\langle q f s, f s\rangle .
\end{gathered}
$$

We now set $\mathscr{D}=\left\{e \in \tilde{E}^{\text {or }} ; e \in \mathcal{O}_{x}\right.$ for some $\left.x \in \mathscr{D}_{V}\right\}$. It is easy to check that $\mathscr{D}$ and $\mathscr{D}=\{\bar{e} ; e \in \mathscr{D}\}$ are fundamental sets in $\tilde{E}^{\text {or }}$ for the natural $\Gamma$-action. 
Note that, if $g_{i}(\sigma e)=\rho(\sigma) g_{i}(e), i=1,2$, for every $\sigma \in \Gamma$ and $e \in \tilde{E}^{\text {or }}$, then the summation

$$
\sum_{e \in \mathscr{Q}}\left\langle g_{1}(e), g_{2}(e)\right\rangle
$$

does not depend on the choice of a fundamental set $\mathscr{D}$. Therefore we find

$$
\begin{aligned}
& \sum_{x \in \mathscr{Q}_{V}}\left\langle\sum_{e \in \mathscr{O}_{x}} f(\mathrm{t}(e))(s(\mathrm{t}(e))-s(x)) w(e), f(x) s(x)\right\rangle \\
& =\sum_{e \in \mathscr{Q}}\langle f(\mathfrak{t}(e))(s(\mathfrak{t}(e))-s(\mathfrak{o}(e))) w(e), f(\mathfrak{o}(e)) s(\mathfrak{o}(e))\rangle \\
& =\sum_{\bar{e} \in \overline{\mathscr{D}}}\langle f(\mathrm{t}(\bar{e}))(s(\mathrm{t}(\bar{e}))-s(\mathfrak{D}(\bar{e}))) w(\bar{e}), f(\mathfrak{o}(\bar{e})) s(\mathfrak{D}(\bar{e}))\rangle \\
& =\sum_{e \in \mathscr{G}}\langle f(\mathfrak{o}(e))(s(\mathfrak{o}(e))-s(\mathfrak{t}(e))) w(e), f(\mathfrak{t}(e)) s(\mathfrak{t}(e))\rangle \\
& =\sum_{x \in \Phi_{V}}\left\langle\sum_{e \in \mathscr{O}_{x}} f(x)(s(x)-s(\mathfrak{t}(e))) w(e), f(\mathfrak{t}(e)) s(\mathfrak{t}(e))\right\rangle,
\end{aligned}
$$

so that

$$
\begin{aligned}
\sum_{x \in \mathscr{Q}_{V}} & \sum_{e \in \mathscr{O}_{x}} f(\mathrm{t}(e)) f(x)\|s(\mathrm{t}(e))-s(x)\|_{W}^{2} w(e) \\
= & \sum_{x \in \mathscr{Q}_{V}} \sum_{e \in \mathscr{o}_{x}}\{\langle f(x)(s(\mathrm{t}(e))-s(x)) w(e), f(\mathrm{t}(e)) s(\mathrm{t}(e))\rangle \\
& \quad-\langle f(x)(s(\mathrm{t}(e))-s(x)) w(e), f(\mathrm{t}(e)) s(x)\rangle\} \\
= & -2 \sum_{x \in \mathscr{Q}_{V}}\left\langle\sum_{e \in \mathscr{O}_{x}} f(\mathrm{t}(e))(s(\mathrm{t}(e))-s(x)) w(e), f(x) s(x)\right\rangle .
\end{aligned}
$$

Combining this with (2.2), we deduce

$$
\frac{\left\langle-\Delta_{\rho} f s, f s\right\rangle+\langle q(f s), f s\rangle}{\|f s\|^{2}}=\lambda_{0}\left(H_{1}\right)+\frac{1}{2} P,
$$

where

$$
P=\frac{\sum_{x \in \mathscr{D}_{V}} \sum_{e \in \mathcal{O}_{x}} f(\mathrm{t}(e)) f(x)\left\|d_{\rho} s(e)\right\|_{W}^{2} w(e)}{\sum_{x \in \mathscr{O}_{V}} f(x)^{2}\|s(x)\|_{W}^{2} m(x)}
$$

There are positive constants $k_{1}, k_{2}$ such that

$$
k_{1} P^{\prime} \leq \inf _{f s \in L_{\rho}^{2}(V)} P \leq k_{2} P^{\prime},
$$

where

$$
P^{\prime}=\frac{\sum_{x \in \mathscr{Q}_{V}} \sum_{e \in \mathscr{O}_{x}}\left\|d_{\rho} s(e)\right\|_{W}^{2} w(e)}{\sum_{x \in \mathscr{D}_{V}}\|s(x)\|_{W}^{2} m(x)} .
$$

Thus, it is enough to show that

$$
c_{1} \delta(\rho, \mathbf{1})^{2} \leq \inf P^{\prime} \leq c_{2} \delta(\rho, \mathbf{1})^{2} .
$$


We now let $\mathscr{U}(\mathscr{D})$ be the set of vertices $x \in \tilde{V}$ such that there exists $e \in \mathscr{D}$ with $\mathrm{t}(e)=x$. It follows from the definition of fundamental set that for every $y \in \mathscr{U}(\mathscr{D})$, there is a unique $\sigma_{y} \in \Gamma$ with $y \in \sigma_{y} \mathscr{D}_{V}$. Consider $B=$ $\left\{\sigma_{y} ; y \in \mathscr{U}(\mathscr{D})\right\} \cup A$, another finite set of generators of $\Gamma$. From the definition of $\delta_{B}(\rho, 1)$, it follows that for every $\varepsilon>0$, there exists a $v \in W$ with $\|v\|=1$ such that $\|\rho(\sigma) v-v\| \leq \delta_{B}(\rho, 1)+\varepsilon$ for all $\sigma \in B$. For this fixed $v$, we define a function $s: V \rightarrow W$ by setting $s(x)=v$ for all $x \in \mathscr{D}_{V}$ and $s(\sigma x)=\rho(\sigma) v$ for every $\sigma x \in \sigma \mathscr{D}_{V}$. It is clear that $s \in L_{\rho}^{2}(V)$. Thus

$$
\sum_{x \in \mathscr{g}_{V}}\|s(x)\|^{2} m(x)=\sum_{x \in \mathscr{g}_{V}} m(x)
$$

and

$$
\sum_{x \in \mathscr{Q}_{V}} \sum_{e \in \mathcal{O}_{x}}\left\|d_{\rho} s(e)\right\|^{2} w(e) \leq\left\{\max _{e \in E} w(e)\right\} \sum_{\sigma \in B}\|\rho(\sigma) v-v\|^{2} \leq C\left\{\delta_{B}(\rho, 1)+\varepsilon\right\}^{2} .
$$

Since $\varepsilon$ is arbitrary, we obtain

$$
\inf P^{\prime} \leq c_{2} \delta(\rho, 1)^{2}
$$

for some positive constant $c_{2}$.

We next show the inequality $c_{1} \delta(\rho, 1)^{2} \leq \inf P^{\prime}$ for some positive constant $c_{1}$. Since for a unit vector $v$,

$$
\delta(\rho, \mathbf{1})^{2} \leq \sum_{\sigma \in A}\|\rho(\sigma) v-v\|^{2},
$$

by substituting $v=s(x) /\|s(x)\|$, we have

$$
\delta(\rho, \mathbf{1})^{2} \sum_{x \in \mathscr{D}_{V}}\|s(x)\|^{2} m(x) \leq \sum_{x \in \mathscr{D}_{V}} \sum_{\sigma \in A}\|s(\sigma x)-s(x)\|^{2} m(x)
$$

for every $s \in L_{\rho}^{2}(V)$. For each $x \in \mathscr{D}_{V}$ and $\sigma \in \Gamma$, we choose a path $C(x, \sigma x)$ in $X$ joining $x$ and $\sigma x$. Let $|C(x, \sigma x)|=\#$ edges in the path $C(x, \sigma x)\}$ and $K=\max _{x \in \mathscr{Q}_{V}} \max _{\sigma \in A}|C(x, \sigma x)|$. The inequality (2.3) and

$$
\|s(\sigma x)-s(x)\|^{2} \leq K \sum_{e \in C(x, \sigma x)}\|s(\mathfrak{t}(e))-s(\mathfrak{D}(e))\|^{2}
$$

imply

$$
\delta(\rho, 1)^{2} \sum_{x \in \mathscr{\Phi}_{V}}\|s(x)\|^{2} m(x) \leq c(\# \mathscr{D}) K^{2} \sum_{x \in \mathscr{\Phi}_{V}} \sum_{e \in \Phi_{x}}\left\|d_{\rho} s(e)\right\|^{2} w(e),
$$

where $c=\max _{x \in V} m(x) \times\left(\min _{e \in E} w(e)\right)^{-1} \times(\# A)$. Thus the proof of the theorem is complete. 


\section{REFERENCES}

[1] R. Brooks, Combinatorial problems in spectral geometry, in the Proc. of Taniguchi Sympo. "Curvature and topology of Riemannian manifolds" 1985, Lecture Notes in Math., 1201, 14-32, Springer, Berlin 1986.

[2] J. Dodziuk and Leon Karp, Spectral and function theory for combinatorial Laplacians, Contemporary Math., 73 (1988), 25-40.

[ 3 ] T. Kobayashi, K. Ono, and T. Sunada, Periodic Schrödinger operators on a manifold, Forum Math., 1 (1989), 69-79.

[4] B. Mohar and W. Woess, A survey on spectra of infinite graphs, Bull. London Math. Soc., 21 (1989), 209-234.

[ 5 ] J. Ortega, Matrix theory a second course, Plenum Press, New York, 1987.

[6] T. Sunada, Fundamental groups and Laplacians, In: Proc. of Taniguchi Symposium on Geometry and Analysis on Manifolds 1987, Lect. Notes in Math., 1339, 248-277, Springer, Berlin 1988.

[ 7 ] _ - Unitary representations of fundamental groups and the spectrum of twisted Laplacians, Topology, 28 (1989), 125-132.

Polly Wee Sy

Department of Mathematics

University of the Philippines

Diliman, Quezon City

Philippines

T. Sunada

Department of Mathematics

University of Tokyo

Tokyo 113

Japan 